\title{
Transcription factor E2-2 inhibits the proliferation of endothelial progenitor cells by suppressing autophagy
}

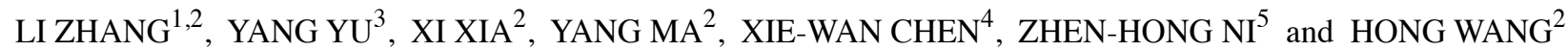 \\ ${ }^{1}$ Department of Postgraduate, Third Military Medical University, Chongqing 400038; \\ ${ }^{2}$ Department of Geriatrics, Kunming General Hospital of Chengdu Military Area, Kunming, Yunnan 650032; \\ ${ }^{3}$ Department of Cardiology, Institute of Cardiovascular Science of PLA; ${ }^{4}$ Cancer Institute of PLA, Xinqiao Hospital, \\ Third Military Medical University; ${ }^{5}$ Department of Biochemistry, Third Military Medical University, Chongqing 400038, P.R. China
}

Received September 12, 2015; Accepted February 24, 2016

DOI: $10.3892 /$ ijmm.2016.2521

\begin{abstract}
Endothelial progenitor cells (EPCs) play a key role in repairing the injured vascular endothelium by differentiating into mature endothelial cells (ECs) or secreting cytokines in a paracrine manner to promote proliferation of existing ECs. However, the mechanisms underlying the proliferation of EPCs were not fully understood. In order to investigate the mechanisms of EPC proliferation, we isolated EPCs from mononuclear cells of mouse spleens. By manipulating E2-2 expression in vitro, we observed that E2-2 negatively regulated the proliferation of EPCs. Moreover, we noted that E2-2 negatively regulated the autophagy of EPCs by studying the expression of LC3II and p62. We also demonstrated that an autophagy inhibitor chloroquine (CQ) decreased the proliferation of EPCs in a concentration-dependent manner. Interestingly, CQ reversed the increase in cell proliferation and autophagy in the E2-2 knockdown group. Furthermore, we detected the expression of autophagy-related protein ATG7 in EPCs which had been transfected with small interfering (siRNA)-E2-2 and siRNA-autophagy related 7 (ATG7) or were untransfected. Our study revealed that E2-2 regulated EPC autophagy via mediating ATG7 expression. We conclude that E2-2 inhibited EPC proliferation via suppressing their autophagy, and E2-2 regulated EPC autophagy by mediating the expression of ATG7.
\end{abstract}

\section{Introduction}

Since the successful isolation of endothelial progenitor cells (EPCs) by Asahara et al in 1997 (1), EPCs have received increasing attention in the field of research on endothelial injury and repair. As the origins of vascular endothelial cells (ECs),

Correspondence to: Professor Hong Wang, Department of Geriatrics, Kunming General Hospital of Chengdu Military Area, 212 Daguan Street, Kunming, Yunnan 650032, P.R. China

E-mail:wangh43@126.com

Key words: E2-2, endothelial progenitor cells, autophagy, proliferation
EPCs repair damaged endothelium by directly differentiating into mature ECs and indirectly secreting paracrine cytokines that promote the proliferation of ECs $(2,3)$. To date, EPC transplantation has been used to treat atherosclerosis and coronary heart disease caused by endothelial injuries (4-6). However, due to the limited proliferative capacity of EPCs, this strategy often leads to insufficient efficacy $(7,8)$. Therefore, enhancing the proliferative ability of EPCs is a key step in improving the effects of EPC transplantation therapy.

The transcription factor E2-2, also referred to as transcription factor 4 (TCF4) or class A basic helix-loop-helix (bHLH) transcription factor, is a member of the E-protein family that is universally expressed in mammalian cells. E2-2 regulates transcription by binding to the E-box binding site in the promoter and enhancer regions of various genes, including nerve-, pancreas- and tumor-specific genes $(9,10)$. It also promotes the proliferation of human liver cancer cells and dermal papilla cells through activating the Wnt/ $\beta$-catenin signaling pathway $(11,12)$. By contrast, another study has shown that knockout of E2-2 increased the proliferative and vessel-forming capacities of mature ECs in mice (13). However, the role and mechanism by which E2-2 regulates the proliferation of EPCs remains unclear.

Autophagy is a highly conserved metabolic process in eukaryotes, and one which is essential for maintaining cellular homeostasis. It has been previously reported that regulation of autophagy affects the proliferation of tumor cells (14), nerve cells (15) and vascular smooth muscle cells (16). A recent study has revealed that inhibition of basal autophagy by 3-methyladenine (3-MA) decreases the growth ability of human EPCs and prevents their differentiation into mature ECs, while hypoxia-induced autophagy increased the growth and differentiation of human EPCs, which led to an increased survival rate of EPCs under hypoxic conditions (17). In recent years, E2-2 has been shown to suppress autophagy by activating the Wnt/ $\beta$-catenin signaling pathway in tumor cells (18). However, whether E2-2 regulates levels of autophagy in EPCs and whether levels of autophagy affect the proliferation of EPCs have not been previously reported.

In the present study, we firstly demonstrated that inhibition of E2-2 expression significantly increased the proliferative ability of EPCs through its effects on autophagy. Moreover, we found 
that E2-2 downregulated the autophagy level by decreasing the expression of AGT7. These findings provide a new insight into EPC proliferation, as we have targeted the 'E2-2/autophagy related 7 (ATG7)/autophagy' pathway, which will benefit EPC transplantation therapy used to treat atherosclerosis and coronary heart disease caused by endothelial injury.

\section{Materials and methods}

Isolation and characterization of EPCs. All animal experiments were approved by the Center of Experimental Animals Committee of Xinqiao Hospital (Chongqing, China). The male C57BL/6J mice (6-8 weeks, 18-20 g, from Xinqiao Hospital Experimental Animal Center, Chongqing, China) were sacrificed by cervical dislocation, and spleens were explanted and thoroughly minced. Spleen-derived mononuclear cells were isolated by density gradient centrifugation (Histopaque 1083; Sigma-Aldrich, St. Louis, MO, USA) at $400 \mathrm{x}$ g for $20 \mathrm{~min}$ in $4^{\circ} \mathrm{C}$. The cells were collected, washed in phosphate-buffered saline (PBS) three times, and then resuspended in Dulbecco's modified Eagle's medium/nutrient mixture F12 (DMEM/F12; Gibco BRL, Gaithersburg, MD, USA) supplemented with $20 \%$ fetal calf serum (FCS; HyClone, Los Angeles, CA, USA), $20 \mathrm{ng} / \mathrm{ml}$ vascular endothelial growth factor (VEGF; R\&D Systems, Inc., Minneapolis, MN, USA), $100 \mathrm{U} / \mathrm{ml}$ penicillin and $100 \mathrm{~g} / \mathrm{ml}$ streptomycin. Cells were seeded into gelatin-coated cell culture flasks and incubated at $37^{\circ} \mathrm{C}$ under an atmosphere with $5 \% \mathrm{CO}_{2}$. Forty-eight hours later, non-attached cells were removed and adherent cells were cultured continuously. Only adherent cells were used in further experiments. The medium was refreshed with complete medium every 2 or 3 days.

To verify the phenotype of EPCs, cells were incubated with Dil-ac-LDL (Biomedical Technologies, Inc., Stoughton, MA, USA) for $3 \mathrm{~h}$, fixed with $4 \%$ paraformaldehyde and then incubated with fluorescein isothiocyanate (FITC)-labeled lectin (UEA-I; Sigma-Aldrich) for $1 \mathrm{~h}$, then washed with PBS three times. The cells were then observed under an immunofluorescence laser scanning confocal microscope (Leica TCS; Leica, Mannheim, Germany). Dual-stained cells positive for Dil-ac-LDL and UEA-I were identified as EPCs. Additionally, fluorescence-activated cell sorting (FACS) analysis was performed using FITC-conjugated antibodies against mouse Sca-1 (ab25031) and vascular endothelial growth factor receptor 2 (VEGFR2; ab11939), and the corresponding isotype control antibodies (ab18446, ab171870; all from Abcam, Cambridge, UK).

Small interfering RNA (siRNA)-mediated silencing of genes. Transient silencing of E2-2 and ATG7 was induced by transfection with siRNAs (siRNA-E2-2 and siRNA-ATG7, respectively) (both from GenePharma, Shanghai, China). The selected siRNA duplex sequences specifically targeted mouse E2-2 and ATG7, and showed no homology to any other sequences during a BLAST search. The siRNA-E2-2 efficient sequence was: sense, 5'-CAAGAAGGAUAUCAAAUCA-3' and antisense, 5'-UGAUUUGAUAUCCUUCUUG-3'. The siRNA-ATG7 sequence was: sense, 5'-GGAGUCACAGCUCUUCCUUTT-3' and antisense, 5'-AAGGAAGAGCUGUGACUCCTT-3'. The corresponding non-silencing control (siRNA-con) sequences (GenePharma) were designed according to the sequence of a negative control. Transfection with siRNA-E2-2 or siRNAATG7 was carried out using Lipofectamine 2000 reagent (Invitrogen, Carlsbad, CA, USA) with a molar ratio between DNA and lipid of approximately 1:3. Forty-eight hours after transfection, cells were collected and used for subsequent assays.

Adenovirus transfection. Adenovirus vector expressing E2-2 (Ad-E2-2) and adenovirus-carrying blank vector (Ad-vector) were both constructed by HanBio Technology Co., Ltd. (Shanghai, China). Before transfection, EPCs were incubated in DMEM/F12 medium without FCS and antibiotics for $6 \mathrm{~h}$. Adenovirus vector was then added to the cells at a multiplicity of infection (MOI) of 100. The transfection medium was removed $2 \mathrm{~h}$ later, and the cells were maintained in the complete medium for $48 \mathrm{~h}$. Ad-vector was used as a control. The mRFP-GFP-LC3 adenovirus vector for detecting autophagy was also purchased from HanBio Technology Co., Ltd. The process of mRFP-GFP-LC3 adenovirus transfection was similar to that of Ad-E2-2. Autophagy was observed under an immunofluorescence laser scanning confocal microscope (Leica TCS). Autophagy was detected by counting the number of green fluorescent protein (GFP) and red fluorescent protein (RFP) dots (dots/cell).

Cell proliferation assay. EPCs were collected from the cultures and replated into a $96-$ well plate $\left(1 \times 10^{5}\right.$ cells $\left./ \mathrm{ml}\right)$ and underwent different treatments. Cell proliferation was measured using a Cell Counting Kit-8 (CCK-8; Beyotime Biotech, Jiangsu, China) according to the manufacturer's instructions. Before reading the absorbance at $450 \mathrm{~nm}, 10 \mu \mathrm{l} \mathrm{CCK}-8$ solution and $100 \mu \mathrm{l}$ complete medium were sequentially added to each well.

In addition, the cell cycle was analyzed by flow cytometric analysis. EPCs were harvested and resuspended in PBS solution at $1 \times 10^{6}$ cells $/ \mathrm{ml}$. The cells were then stored in $70 \%$ ethanol at $4^{\circ} \mathrm{C}$ overnight. Before being analyzed, the cells were mixed with $1 \mathrm{ml}$ propidium iodide (PI; $1 \mathrm{mg} / \mathrm{ml}$ ) (BD Biosciences, Bedford, MA, USA) and then incubated at $4^{\circ} \mathrm{C}$ for $30 \mathrm{~min}$. After centrifugation, the cells were resuspended and the cell cycle was analyzed immediately using a flow cytometer (MoFloTMXDP; Beckman Coulter, Co. Fullerton, CA, USA). The proliferation index was defined as the total percentage of cells in $\mathrm{S}$ and $\mathrm{G} 2 / \mathrm{M}$ phases. All groups of experiments were undertaken in triplicate.

Western blot analysis. EPCs were lysed in ice-cold lysis buffer (RIPA) containing a protease inhibitor (0.5 mM PMSF) (both from Beyotime Biotech). The cell lysates were further centrifuged at $15,000 \mathrm{x}$ g for $15 \mathrm{~min}$ at $4^{\circ} \mathrm{C}$. Proteins from EPC lysates were measured using the Bradford method. The same amount of protein was loaded in each lane, separated by $10-15 \%$ sodium dodecyl sulfate-polyacrylamide gel electrophoresis (SDS-PAGE), and transferred to $0.22 \mu \mathrm{m}$ polyvinylidene difluoride (Millipore Corp., Boston, MA, USA) membranes. Membranes were blocked with 5\% non-fat milk $2 \mathrm{~h}$ followed by incubated with primary antibodies overnight at $4^{\circ} \mathrm{C}$. The primary antibodies included: rabbit anti-mouse LC3 polyclonal antibody (1:1,000; Cat. no. 2775), rabbit anti-mouse p62 monoclonal antibody $(1: 1,000$; Cat. no. 5114), rabbit antimouse ATG7 polyclonal antibody $(1: 1,000$; Cat. no. 8558) 

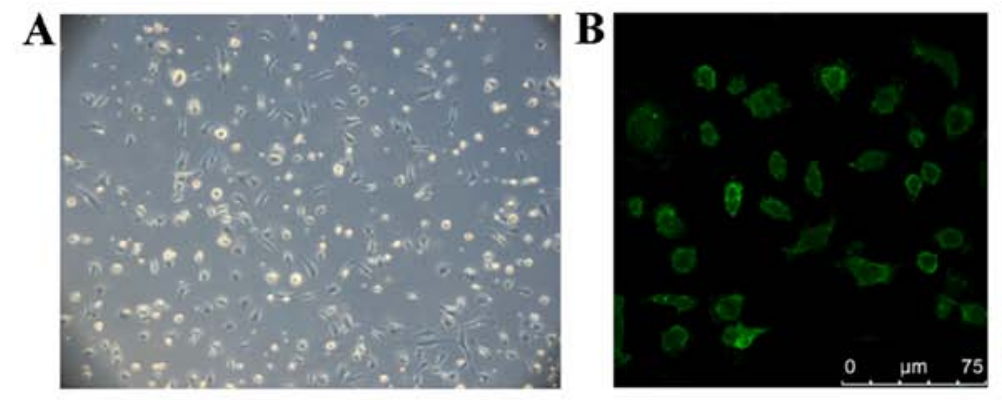

FITC-UEA-I

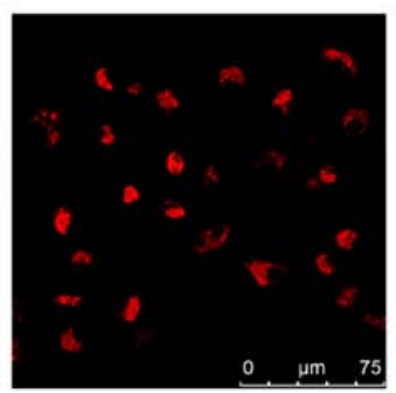

DiI-ac-LDL

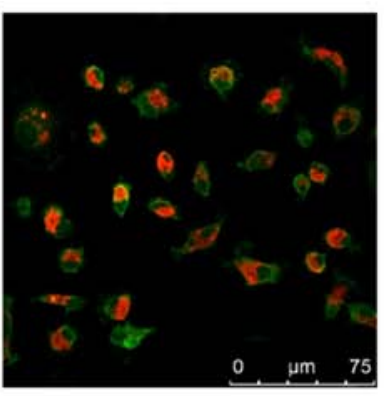

Merge

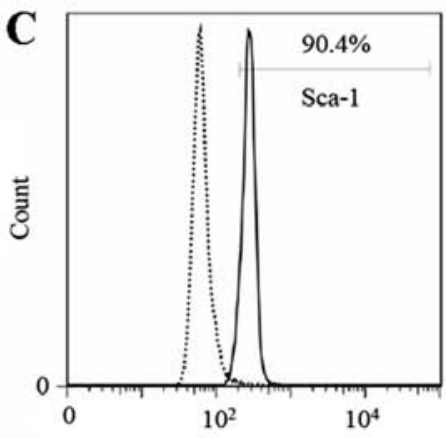

FL1-H

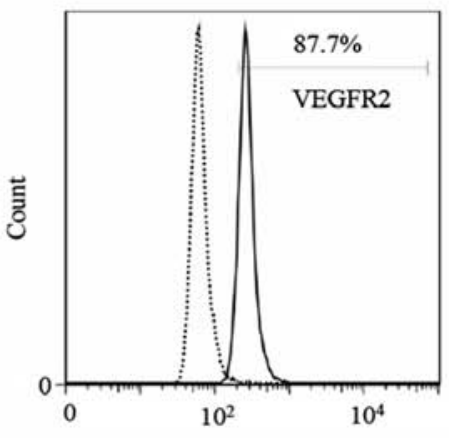

FL1-H

Figure 1. Properties of mouse spleen-derived endothelial progenitor cells (EPCs) after in vitro 7-day culture. (A) Morphology of mouse EPCs after 7-day culture. The mouse EPCs displayed spindle and oval shapes under a microscope (x100). (B) Immunocytochemical analysis of EPCs with FITC-UEA-I and Dilac-LDL. EPCs are stained with FITC-UEA-I (green) and DiI-ac-LDL (red). The percentage of double-positive cells was $92.33 \pm 2.63 \%$. (n=3; scale bar, $75 \mu \mathrm{m}$ ). (C) Representative images of FACS analysis of cultured EPCs. The percentage of Scal-1+ and VEGFR2 ${ }^{+}$cells was 90.4 and $87.7 \%$, in the right area of the box, respectively. The dotted lines in the left area of each box represent corresponding negative isotype control labeling, and the line denotes a positive gate.

(all from Cell Signaling Technology, Inc., Boston, MA, USA) and rat anti-mouse $\beta$-actin monoclonal antibody $(1: 1,000$; Cat. no. AA128; Beyotime Biotech). The membranes were then incubated with horseradish peroxidase (HRP)-conjugated secondary antibodies (1:5,000; A0208, A0216; Beyotime Biotech) for $1 \mathrm{~h}$ at $37^{\circ} \mathrm{C}$. Protein bands were visualized by enhanced chemiluminescence (ECL) (Thermo Fisher Scientific, Waltham, MA, USA), and densitometric signals were quantified using ImageQuant TL software (General Electric, Co., Fairfield, CT, USA).

RNA isolation and reverse transcription-quantitative polymerase chain reaction ( $R T-q P C R)$. Total RNA was isolated from EPCs using RNAiso Plus (Takara Bio, Dalian, China) according to the manufacturer's instructions. Single cDNAs was synthesized from RNA using a PrimeScript ${ }^{\mathrm{TM}} \mathrm{RT}$ reagent kit (Takara Bio). The following primer sequences were used for ATG7 (sense, 5'-ACCCAGAAGAAGCTGAACGA-3', and antisense, 5'-CTCATTTGCTGCTTGTTCCA-3'); $\beta$-actin (sense, 5'-TTCTACAATGAGCTGCGTGTGG-3' and antisense, 5'-GTGTTGAAGGTCTCAAACATGAT-3'). SYBR ${ }^{\circledR}$ Premix Ex Taq ${ }^{\mathrm{TM}}$ II (Takara Bio) was used according to the manufacturer's instructions, and RT-qPCR was performed using a StepOnePlus ${ }^{\mathrm{TM}}$ Real-Time PCR system (Applied Biosystems Life Technologies, Waltham, MA, USA). The reaction conditions were as follows: $95^{\circ} \mathrm{C}$ for $30 \mathrm{sec}, 40$ cycles of $95^{\circ} \mathrm{C}$ for $5 \mathrm{sec}$, and $60^{\circ} \mathrm{C}$ for $30 \mathrm{sec}$. Relative expression of target mRNA was normalized to $\beta$-actin using the $\Delta \Delta \mathrm{Ct}$ method, as previously described (19).
Statistical analysis. Data from at least three independent experiments are expressed as the means \pm SD. SPSS 16.0 software was used for statistical analysis. Student's t-tests were used for comparisons between groups. A P-value $<0.01$, or $\mathrm{P}<0.05$ was considered to indicate a statistically significant difference.

\section{Results}

Biological properties of EPCs. After 7 days of being cultured, round, oval and spindle shapes of attached spleen-derived mononuclear cells were noted under the microscope (Fig. 1A), similar to morphologies which have been previously described (20). Immunocytochemical (ICC) staining demonstrated that $92.33 \pm 2.63 \%(\mathrm{n}=3)$ of attached cells were stained positively for FITC-UEA-I and Dil-ac-LDL (Fig. 1B), validating the theory that the FITC-UEA-I/Dil-ac-LDL double-positive cells possessed the characteristics of ECs. Furthermore, the attached cells were identified by analyzing the expression of the mouse stem-cell marker Sca-1 and EC marker VEGFR2 using flow cytometry. The percentage of positive cells was $88.93 \pm 1.31$ and $85.13 \pm 2.83 \%$ ( $n=3$ ), respectively (Fig. 1C). Taking these results into account, the attached cells were considered EPCs.

E2-2 inhibits the proliferation of EPCs. To determine whether E2-2 regulated the proliferation of EPCs, we overexpressed or silenced E2-2 by transfecting Ad-E2-2 or siRNA-E2-2 into the cells, and proliferation was then detected by CCK- 8 and cell cycle assays in vitro. The CCK- 8 assay demonstrated that the proliferation of EPCs significantly decreased in the E2-2 
A

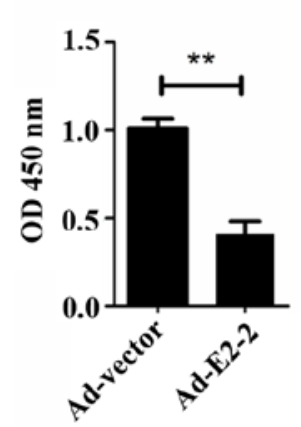

D

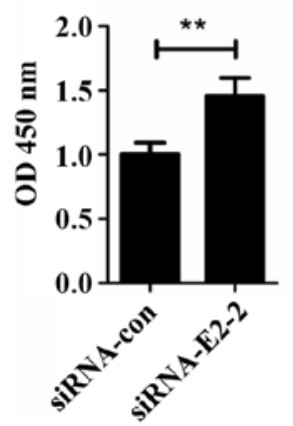

B

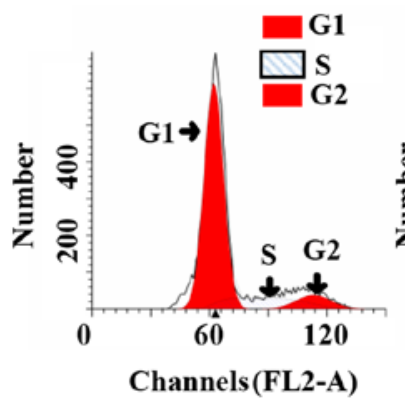

$\mathbf{E}$

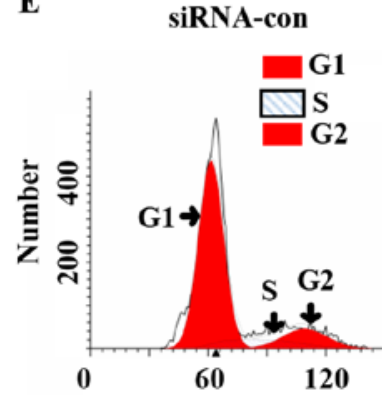

Channels (FL2-A)

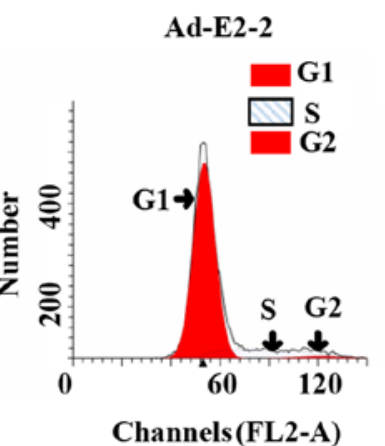

C

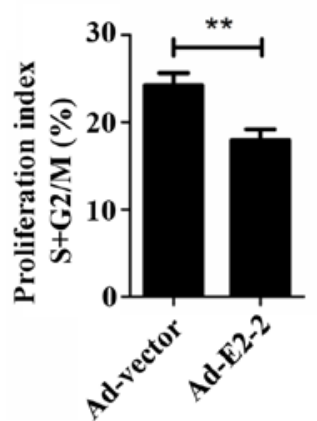

$\mathbf{F}$

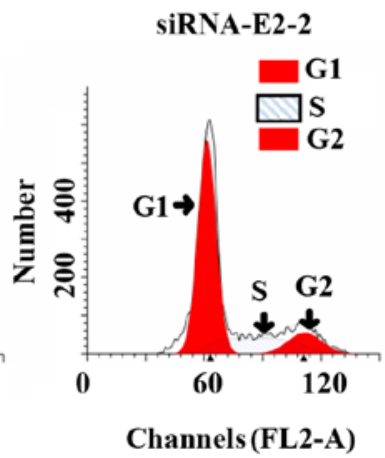

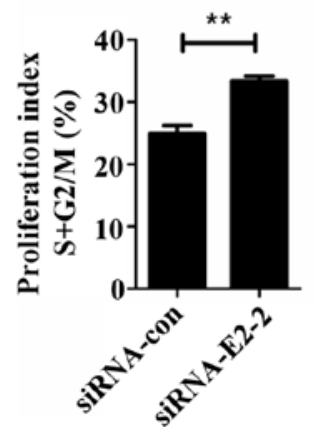

Figure 2. E2-2 inhibits the proliferation of endothelial progenitor cells (EPCs) in vitro. (A) A CCK-8 assay was used to measure the proliferation of EPCs transfected with adenovirus vector expressing E2-2 (Ad-E2-2) and Ad-vector at 100 multiplicity of infection (MOI) for $48 \mathrm{~h}$. The OD value significantly decreased in the Ad-E2-2 group compared with the Ad-vector group $\left({ }^{* *} \mathrm{P}<0.01, \mathrm{n}=3\right.$ ). (B) Representative images of flow cytometry showed that the percentage of cells in S and G2/M phases was lower in the Ad-E2-2 group than in the Ad-vector group. (C) The bar diagram represents results of the cell cycle analysis. The proliferation index in the Ad-E2-2 group significantly decreased compared with the Ad-vector group (** $\mathrm{P}<0.01, \mathrm{n}=3$ ). (D) The CCK-8 assay revealed that the OD value of EPCs transfected with siRNA-E2-2 at a concentration of $50 \mathrm{nM}$ for $48 \mathrm{~h}$ increased significantly compared with the siRNA-con group ( ${ }^{* *} \mathrm{P}<0.01$, $\mathrm{n}=3$ ). (E) Flow cytometric representative images showed that the percentage of cells in the $\mathrm{S}$ and $\mathrm{G} 2 / \mathrm{M}$ phases in the siRNA-E2-2 group was higher than that in the siRNA-con group. (F) The statistical results of cell cycle analysis revealed that the proliferation index in the siRNA-E2-2 group significantly increased compared with the siRNA-con group $(" \mathrm{P}<0.01, \mathrm{n}=3)$.

overexpression (Ad-E2-2) group compared with the adenoviral control vector (Ad-vector) group (Fig. 2A). Cell cycle analysis showed that the proliferation index $(\mathrm{S}+\mathrm{G} 2 / \mathrm{M})$ of the EPCs was lower in the Ad-E2-2 group than in the Ad-vector group (Fig. 2B and C). The trend of the cell cycle was similar to the results of the CCK-8 assay. However, in the knockdown E2-2 (siRNA-E2-2) group, the proliferative capacity of EPCs significantly increased compared with that in the negative control (siRNA-con) group (Fig. 2D). The EPC proliferation index $(\mathrm{S}+\mathrm{G} 2 / \mathrm{M})$ of the siRNA-E2-2 group was higher than that of the siRNA-con group (Fig. 2E and F). These results suggest that E2-2 suppressed the proliferation of EPCs in vitro.

E2-2 negatively regulates the autophagy of EPCs. To investigate whether E2-2 regulates autophagy in EPCs, we firstly studied the expression of LC3II and p62 while overexpressing or knocking down E2-2. We noted a significant decrease in LC3II expression, but a marked increase in the bands of p62, in the Ad-E2-2 group compared with the Ad-vector group (Fig. 3A), suggesting that overexpression of E2-2 induced suppression of autophagy in EPCs. By contrast, LC3II expression increased and the protein level of p62 decreased in the knockdown E2-2 group (Fig. 3B). Thus, knockdown of E2-2 upregulated autophagy of EPCs. In addition, we used the mRFP-GFP-LC3 adenovirus to confirm the regulatory effects of E2-2 on autophagy. This assay is used to monitor progression from autophagosome to autolysosome based on the $\mathrm{pH}$ difference between the acidic autolysosome and the neutral autophagosome and the $\mathrm{pH}$ sensitivity differences displayed by GFP and RFP. When autolysosomes are formed by fusing autophagosome with a lysosome, the GFP moiety degrades from the tandem protein, but RFP-LC3 maintains the dots $(21,22)$. The red dots indicate autophagosomes, and yellow dots (merge of RFP and GFP) represent autolysosomes. When we co-transfected mRFP-GFP-LC3 adenovirus with Ad-E2-2 or siRNA-E2-2 in EPCs for $48 \mathrm{~h}$, we observed that overexpression of E2-2 reduced the numbers of autophagosomes and autolysosomes (Fig. 3C). Conversely, knockdown of E2-2 increased not only the number of autophagosomes but also that of autolysosomes (Fig. 3D). The results further confirmed that E2-2 negatively regulated autophagy of EPCs.

E2-2 inhibitstheproliferationofEPCsviasuppressingautophagy. To validate the role autophagy plays in EPC proliferation, we treated EPCs with chloroquine (CQ) at different concentrations and different periods of time. It was observed that CQ inhibited the proliferation of EPCs in a concentration-dependent manner. After treatment with CQ for $24 \mathrm{~h}$ at $20 \mu \mathrm{M}$, the proliferation of EPCs as measured by CCK- 8 decreased markedly compared with the group which was not treated with CQ, and the degree of decrease changed slightly with the increasing periods of 
A
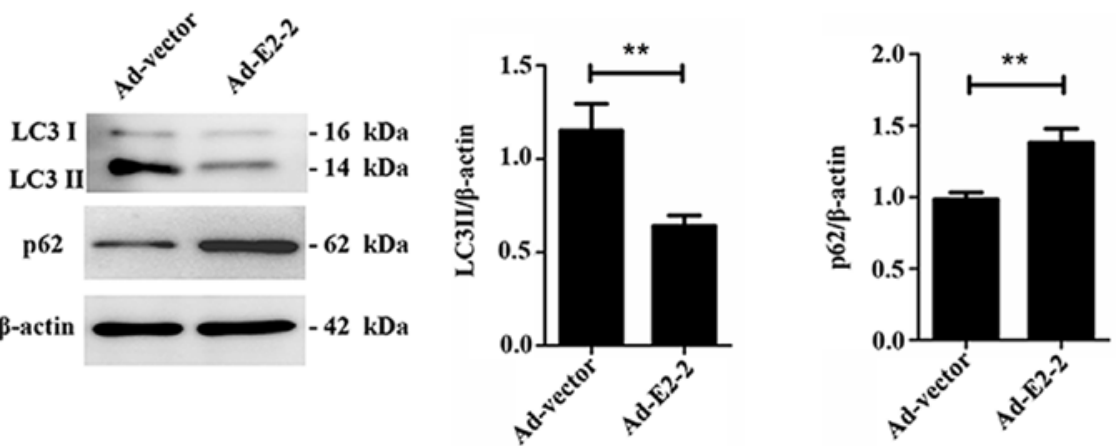

B
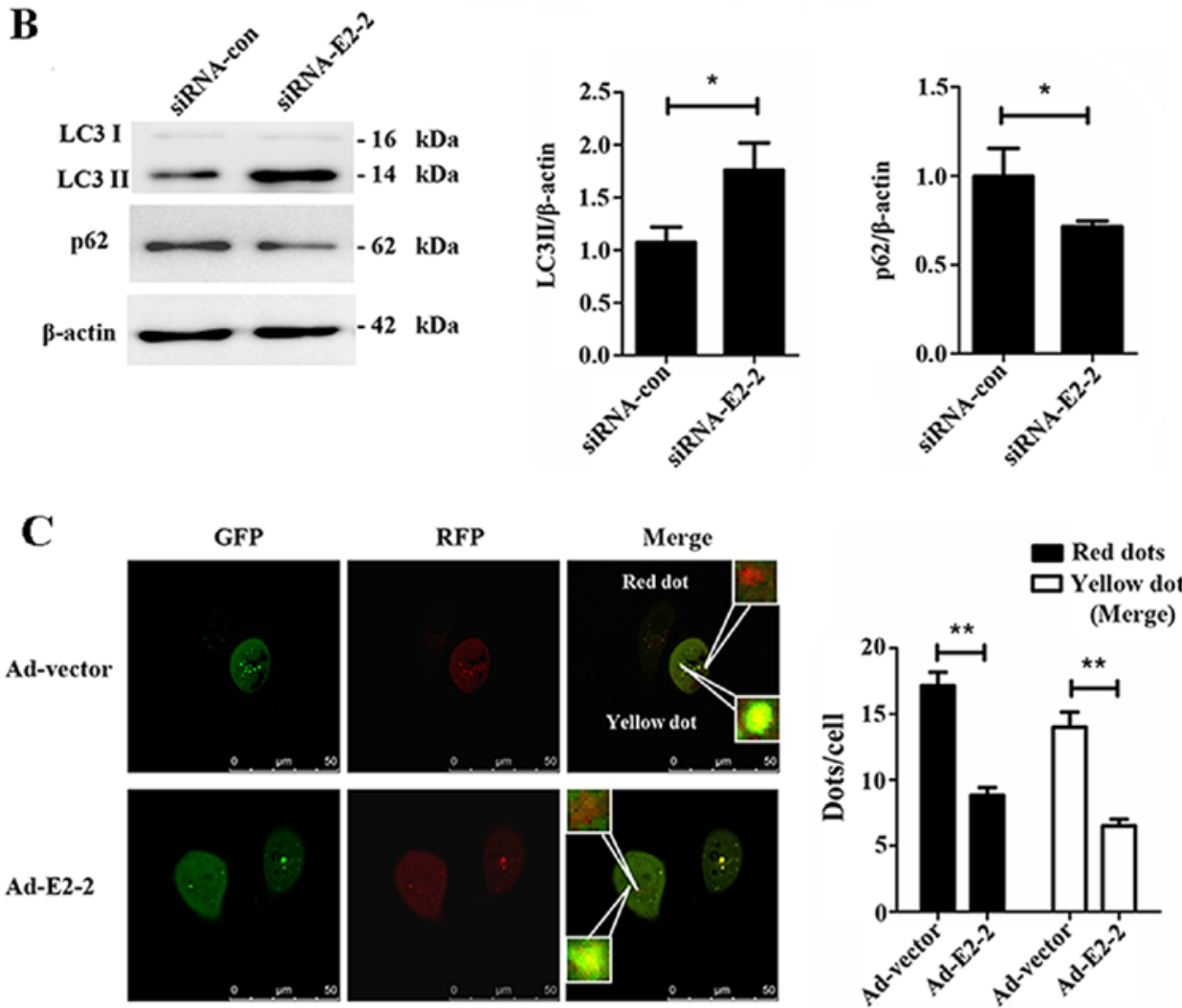

Ad-E2-2
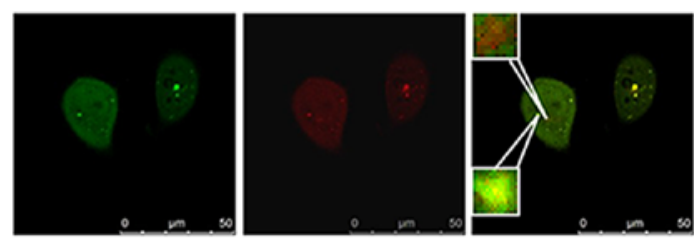

RFP
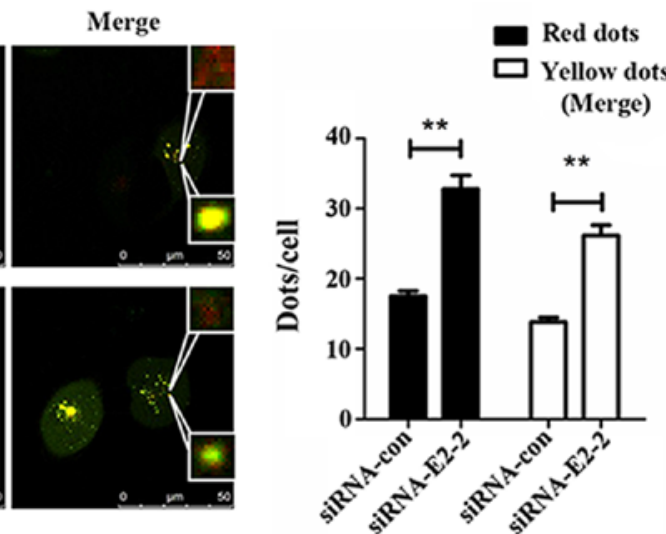

Figure 3. E2-2 negatively regulates the autophagy of endothelial progenitor cells (EPCs). (A) Representative images of western blot analysis and summarized data showing the levels of autophagy-related proteins in EPCs transfected with adenovirus vector expressing E2-2 (Ad-E2-2) and Ad-vector. In the Ad-E2-2 group, LC3II expression decreased and p62 expression increased significantly compared with the Ad-vector group $\left({ }^{* *} \mathrm{P}<0.01, \mathrm{n}=3\right)$. (B) Representative images of western blot analysis and summarized data showing the levels of autophagy-related proteins in EPCs transfected with siRNA-E2-2 and siRNA-con. The protein level of LC3II was higher while p62 was lower in the siRNA-E2-2 group than in the siRNA-con group ( $\mathrm{P}<0.05, \mathrm{n}=3$ ). (C) Representative images from the immunofluorescence laser scanning confocal microscope and statistical data gained by transfection with Ad-E2-2 or Ad-vector for $48 \mathrm{~h}$. The red and yellow dots per cell significantly decreased in Ad-E2-2 group compared with Ad-vector group $\left({ }^{* *} \mathrm{P}<0.01, \mathrm{n}=3\right.$; scale bar, $\left.50 \mu \mathrm{m}\right)$. (D) Representative images and statistical data gained by co-transfection with siRNA-E2-2 or siRNA-con for $48 \mathrm{~h}$. The number of red and yellow dots per cell in the siRNA-E2-2 group significantly increased compared with the siRNA-con group $(* * \mathrm{P}<0.01, \mathrm{n}=3$; scale bar, $50 \mu \mathrm{m})$. 
A

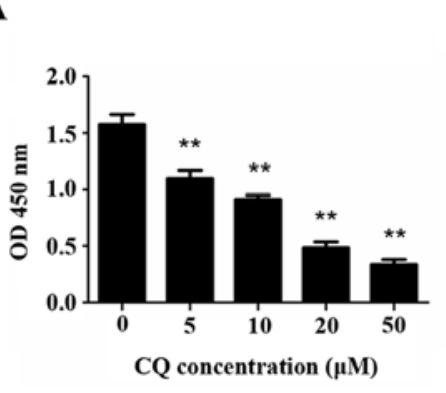

B

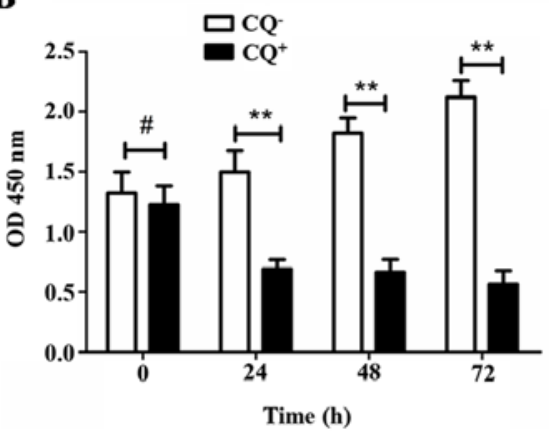

C

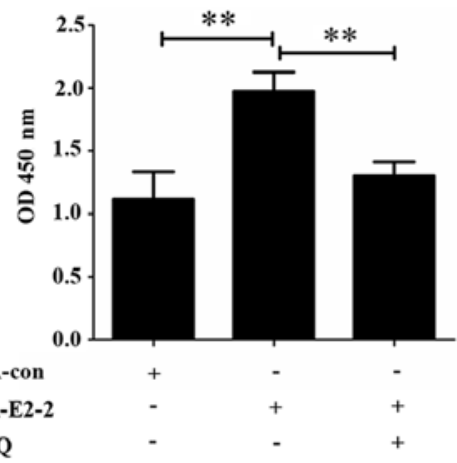

CQ

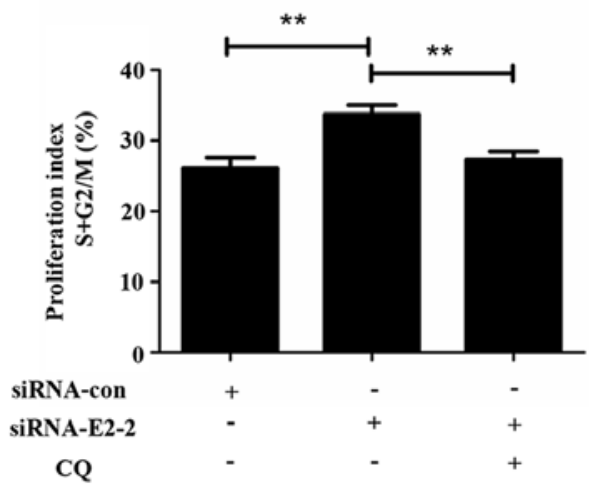

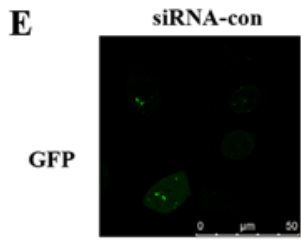

D

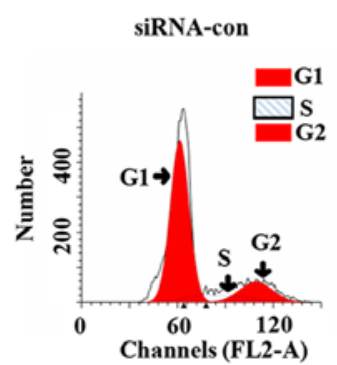

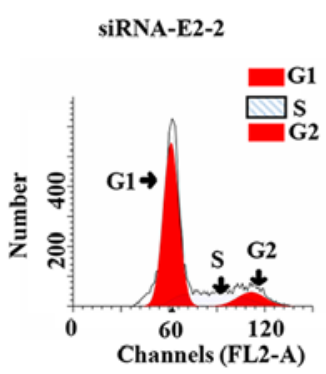

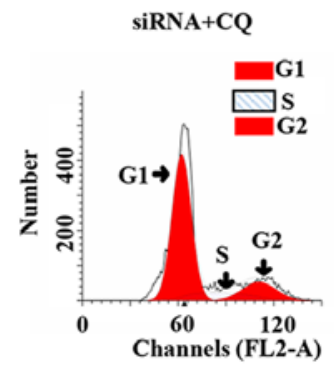

SiRNA-E2-2
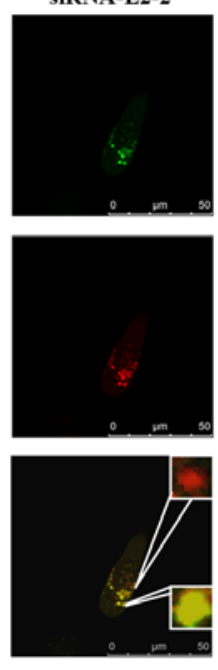
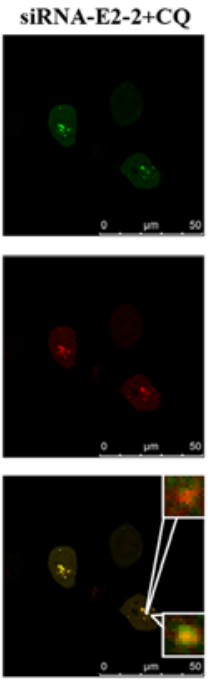
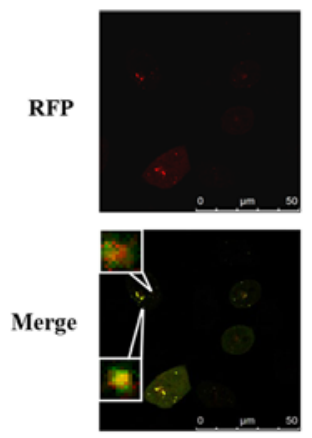

Figure 4. E2-2 inhibits the proliferation of endothelial progenitor cells (EPCs) via suppressing autophagy. (A) Chloroquine (CQ) was used to treat EPCs for $24 \mathrm{~h}$. A CCK-8 assay showed that the OD value significantly decreased at 5, 10, 20 and $50 \mu \mathrm{M}\left({ }^{* *} \mathrm{P}<0.01, \mathrm{n}=3\right)$. (B) CQ-treated EPCs at $20 \mu \mathrm{M}$ for different times. In the groups treated with CQ for 24,48 and $72 \mathrm{~h}$, the absorbance of EPCs was significantly reduced compared with that in CQ-free group ("* $\mathrm{P}<0.01, \mathrm{n}=3$ ). No significant difference was observed in absorbance between CQ-treated and CQ-free groups at $0 \mathrm{~h}\left({ }^{*} \mathrm{P}>0.05, \mathrm{n}=3\right)$. (C) Absorbance at $450 \mathrm{~nm}$ was detected by CCK-8 assay after $48 \mathrm{~h}$ treatment of EPCs with siRNA-con, siRNA-E2-2, and siRNA-E2-2+CQ. The OD value was significantly higher in the siRNA-E2-2 group than in siRNA-con and siRNA+CQ groups $\left({ }^{* *} \mathrm{P}<0.01, \mathrm{n}=3\right)$. (D) Representative images of flow cytometry and statistical data were indicative of distinct increases in proliferation index $(\mathrm{S}+\mathrm{G} 2 / \mathrm{M})$ in the siRNA-E2-2 group compared with the siRNA-con and siRNA+CQ groups ("* $\mathrm{P}<0.01, \mathrm{n}=3)$. (E) Representative images of immunofluorescence laser scanning using a confocal microscope and statistical data from different groups. The numbers of red and yellow dots per cell were significantly higher in the siRNA-E2-2 group compared to the siRNA-con and siRNA-E2-2+CQ groups ( ${ }^{* *} \mathrm{P}<0.01, \mathrm{n}=3 ; \mathrm{scale}$ bar, $\left.50 \mu \mathrm{m}\right)$.

time (Fig. 4B). Subsequently, to determine whether autophagy was involved in E2-2-mediated EPC proliferation, we added $20 \mu \mathrm{M}$ CQ to EPCs after transfection with siRNA-E2-2 for $24 \mathrm{~h}$. After transfection for $48 \mathrm{~h}$, the CCK-8 assay showed that the increase in proliferation in the siRNA-E2-2 group was partially reversed by the administration of CQ (Fig. 4C). The alteration of the proliferation index, as measured by flow cytometry, was similar to the results of the CCK-8 assay (Fig. 4D). Furthermore, we transfected the different groups of EPCs with siRNA-con, siRNA-E2-2 or siRNA-E2-2 and CQ and found that CQ inhibited the increase in autophagosomes and autolysosomes (Fig. 4E), suggesting that EPC autophagy induced by E2-2 knockdown was reversed by CQ. These results demonstrated that inhibition of basal autophagy reduced the proliferation of EPCs, and E2-2 inhibited the proliferation of EPCs through its effects on autophagy. 


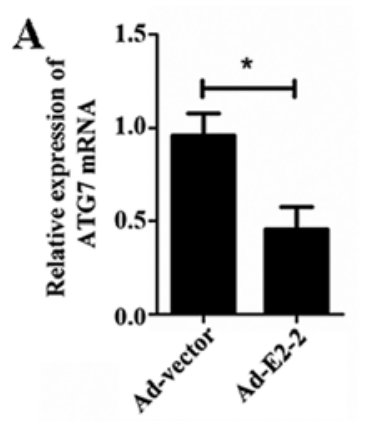

B
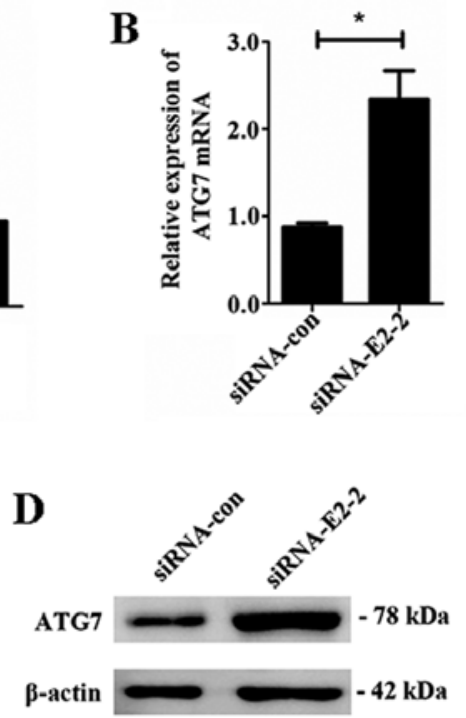

$\mathbf{E}$

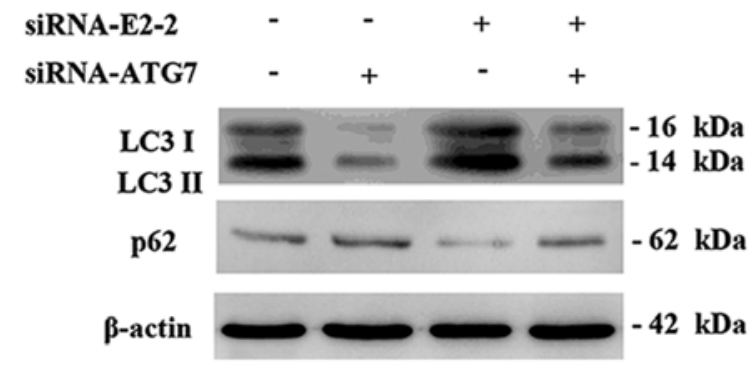

C
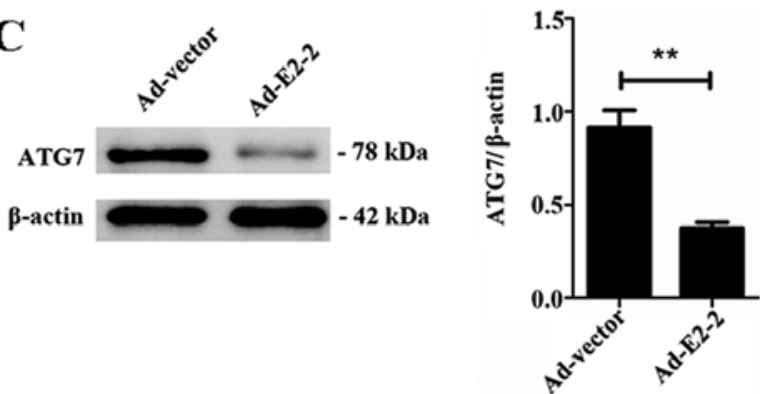
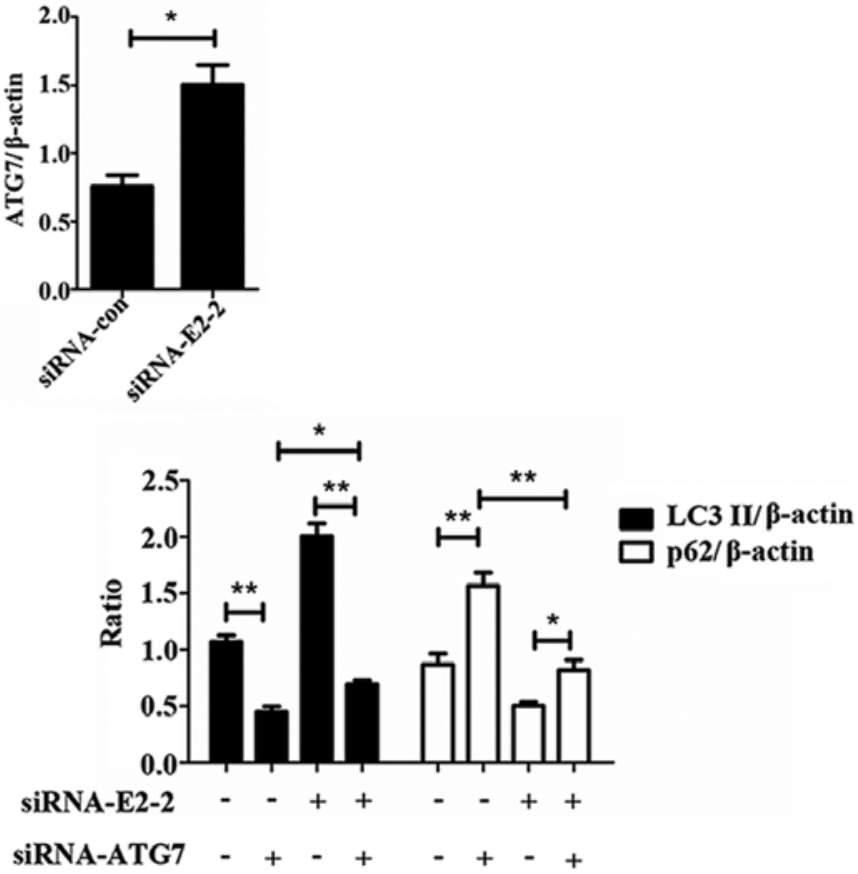

Figure 5. E2-2 regulates autophagy of endothelial progenitor cells (EPCs) via mediating the expression of autophagy related 7 (ATG7). (A) Quantitative analysis of ATG7 mRNA expression by RT-qPCR after transfection with adenovirus vector expressing E2-2 (Ad-E2-2) and Ad-vector for $48 \mathrm{~h}$. The mRNA expression of ATG7 was lower in the Ad-E2-2 group than in the Ad-vector group ( ${ }^{*} \mathrm{P}<0.05, \mathrm{n}=3$ ). (B) Quantitative analysis of ATG7 mRNA expression by RT-qPCR after transfection with siRNA-E2-2 and siRNA-con for $48 \mathrm{~h}$. mRNA expression of ATG7 increased in siRNA-E2-2 group compared with siRNA-con group ( $\mathrm{P}<0.05, \mathrm{n}=3)$. (C) Representative images of western blot analysis and summarized data showing protein expression of ATG7 after transfection with Ad-E2-2 and Ad-vector. Protein expression of ATG7 significantly decreased in Ad-E2-2 compared with Ad-vector group ( ${ }^{* * *} \mathrm{P}<0.01, \mathrm{n}=3$ ). (D) Representative images of western blot analysis and summarized data showing the protein expression of ATG7 after transfection with siRNA-E2-2 and siRNA-con. Protein expression of ATG7 was significantly increased in the siRNA-E2-2 compared with the siRNA-con group ( $\mathrm{P}<0.05, \mathrm{n}=3$ ). (E) Representative images of western blot analysis and summarized data showing protein expression of LC3II and p62 after co-transfection or respective transfection with siRNA-E2-2 and siRNA-ATG7 $\left({ }^{* *} \mathrm{P}<0.01,{ }^{*} \mathrm{P}<0.05, \mathrm{n}=3\right)$.

E2-2 regulates autophagy of EPCs via mediating the expression of ATG7. To further explore the mechanism by which E2-2 regulates autophagy in EPCs, we detected the expression of the autophagy-related gene ATG7 after E2-2 overexpression or knockdown. We found that overexpression of E2-2 decreased the expression level of ATG7 mRNA (Fig. 5A), and knockdown of E2-2 upregulated the expression of ATG7 mRNA (Fig. 5B). Subsequently, we noted that overexpression of E2-2 downregulated ATG7 protein expression, and knockdown of E2-2 upregulated ATG7 protein expression (Fig. 5C and D). Furthermore, to demonstrate whether ATG7 was involved in E2-2-mediated autophagy, we transfected the cells with siRNA-E2-2 and siRNA-ATG7 simultaneously or respectively. The transfection revealed that autophagy of EPCs was downregulated by ATG7 knockdown alone, and the increased autophagy induced by siRNA-E2-2 was reversed by ATG7 knockdown. Interestingly, the level of autophagy was still higher in the co-transfected siRNA-E2-2 and siRNA-ATG7 group than in siRNA-ATG7 group (Fig. 5E). We demonstrated that E2-2 regulated autophagy of EPCs via partially mediating the expression of ATG7.

\section{Discussion}

As a transcription factor, E2-2 binds to the promoter regions and affects the expression of target proteins, ultimately influencing several cellular biological functions, including proliferation. In ECs, Bach1 binds to E2-2, which represses the activation of Wnt/ $\beta$-catenin signaling and decreases the proliferative and vessel-forming abilities of the cells (23). Tanaka et al revealed that E2-2 suppressed the proliferation of vascular ECs via 
binding to the promoter of VEGFR2. Knockdown of the E2-2 gene significantly improved the proliferative and vessel-forming abilities of ECs (13). Consistent with these reports, our findings showed that E2-2 negatively regulated the proliferation of EPCs. However, another study has shown that downregulation of E2-2 suppressed the proliferation and survival of corneal epithelial stem cells (24). Xiong et al demonstrated that upregulation of E2-2 activated the Wnt/ $\beta$-catenin signaling pathway, initiating the proliferation of dermal papilla cells (12). Therefore, we consider that the mechanisms of E2-2 in the regulation of cell proliferation are complicated, and may be associated with the different cellular contents and microenvironment.

Autophagy is a highly conserved cell activity that involves degradation of dysfunctional organelles and proteins. The breakdown of cellular components promotes cellular survival by maintaining cellular energy levels and homeostasis $(25,26)$. Previously it has been confirmed that autophagy plays an indispensable role in maintaining stem-cell characteristics, self-renewal, and the capacity for differentiation in various stem cells (27). Moreover, previous research has demonstrated that upregulation of autophagy promotes the proliferation of cancer cells (28), while downregulation of autophagy decreases cell proliferation (29). In nerve cells, autophagy induced by high mobility group box 1 protein (HMGB1) increased the growth of neuroblasts (30). In immune cells, inhibition of autophagy decreased cell growth and differentiation and promoted apoptosis (31). By contrast, another study has suggested that induction of autophagy suppressed cancer cell proliferation (32). In the present study, inhibition of autophagy at basal levels by the inhibitor CQ decreased the proliferative ability of EPCs. Therefore, we suggest that distinct cell types have different levels of autophagy, thus generating various effects on cell growth and survival. The underlying mechanism of action remains to be explored.

In the present study, to the best of our knowledge, we were the first to find that E2-2 inhibited the autophagy of EPCs via decreasing the expression of ATG7. ATG7 is a gene that plays a key role in autophagy and normal cell physiology $(33,34)$. Previous studies showed that ATG7 is required for normal functioning of ECs via maintaining the basal levels of autophagy (35). In HeLa cells, inhibition of ATG7 suppressed the level of autophagy, which decreased cell proliferation (36). Moreover, Lee et al discovered that depletion of ATG7 suppressed autophagy and inhibited the activation of p53 in mouse embryonic fibroblasts, thus inducing cell cycle arrest and apoptosis (37). Our present study is thus the first to elucidate that overexpression of E2-2 in mouse EPCs downregulated ATG7 mRNA and protein levels, whereas knockdown of E2-2 led to upregulation of ATG7 mRNA and protein levels. After simultaneous or respective knockout of E2-2 and ATG7 with siRNAs, we found that knockdown of ATG7 caused a decrease in autophagy levels. Moreover, increased autophagy levels after knockdown of E2-2 waspartially reversed by ATG7 knockdown. Our findings suggest that in EPCs ATG7 mRNA and protein levels are regulated by E2-2. Increased levels of autophagy after depletion of E2-2 may be partially attributed to the enhanced transcription of ATG7.

Therefore, we noted the existence of the E2-2/ATG7/autophagy signaling pathway and its regulatory effects on EPC proliferation. However, the binding site of E2-2 with ATG7 and the mechanism by which ATG7 transcription is regulated remain to be explored in future research.

The present study attempted to clarify the mechanism underlying EPC proliferation by investigating autophagy, and provided new insights into research on EPCs and potential novel therapies for repairing endothelial injury and treating atherosclerosis and coronary heart disease.

\section{Acknowledgements}

The present study was supported by grants from the National Natural and Science Foundation of China(NSFC) (no.81270224). We thank Ms. Huali Kang, Ms. Chunxing Xu and Mr. Jie Yang for their kind help (Department of Cardiology, Institute of Cardiovascular Science of PLA, Xinqiao Hospital, Third Military Medical University). Thanks to Professor Jihang Zhang (Department of Cardiology, Institute of Cardiovascular Science of PLA, Xinqiao Hospital, Third Military Medical University) for a critical reading of the manuscript.

\section{References}

1. Asahara T, Murohara T, Sullivan A, Silver M, van der Zee R, Li T, Witzenbichler B, Schatteman G and Isner JM: Isolation of putative progenitor endothelial cells for angiogenesis. Science 275: 964-967, 1997.

2. Ii M, Nishimura H, Iwakura A, Wecker A, Eaton E, Asahara T and Losordo DW: Endothelial progenitor cells are rapidly recruited to myocardium and mediate protective effect of ischemic preconditioning via 'imported' nitric oxide synthase activity. Circulation 111: 1114-1120, 2005.

3. George AL, Bangalore-Prakash P, Rajoria S, Suriano R, Shanmugam A, Mittelman A and Tiwari RK: Endothelial progenitor cell biology in disease and tissue regeneration. J Hematol Oncol 4: 24, 2011.

4. Wassmann S, Werner N, Czech T and Nickenig G: Improvement of endothelial function by systemic transfusion of vascular progenitor cells. Circ Res 99: e74-e83, 2006.

5. Lee PS and Poh KK: Endothelial progenitor cells in cardiovascular diseases. World J Stem Cells 6: 355-366, 2014.

6. Xu JY, Lee YK, Wang Y and Tse HF: Therapeutic application of endothelial progenitor cells for treatment of cardiovascular diseases. Curr Stem Cell Res Ther 9: 401-414, 2014.

7. Wöhrle J, Birkemeyer R, Markovic S, Nguyen TV, Sinha A, Miljak T, Spiess J, Rottbauer W and Rittger H: Prospective randomised trial evaluating a paclitaxel-coated balloon in patients treated with endothelial progenitor cell capturing stents for de novo coronary artery disease. Heart 97: 1338-1342, 2011.

8. Raval Z and Losordo DW: Cell therapy of peripheral arterial disease: from experimental findings to clinical trials. Circ Res 112: 1288-1302, 2013.

9. Massari ME and Murre C: Helix-loop-helix proteins: regulators of transcription in eucaryotic organisms. Mol Cell Biol 20: 429-440, 2000

10. Sobrado VR, Moreno-Bueno G, Cubillo E, Holt LJ, Nieto MA, Portillo F and Cano A: The class I bHLH factors E2-2A and E2-2B regulate EMT. J Cell Sci 122: 1014-1024, 2009.

11. Zhao DH, Hong JJ, Guo SY, Yang RL, Yuan J, Wen CY, Zhou KY and Li CJ: Aberrant expression and function of TCF4 in the proliferation of hepatocellular carcinoma cell line BEL-7402. Cell Res 14: 74-80, 2004.

12. Xiong Y, Liu Y, Song Z, Hao F and Yang X: Identification of Wnt/ $/$-catenin signaling pathway in dermal papilla cells of human scalp hair follicles: TCF4 regulates the proliferation and secretory activity of dermal papilla cell. J Dermatol 41: 84-91, 2014.

13. Tanaka A, Itoh F, Nishiyama K, Takezawa T, Kurihara H, Itoh S and Kato M: Inhibition of endothelial cell activation by bHLH protein E2-2 and its impairment of angiogenesis. Blood 115: 4138-4147, 2010.

14. Guo S, Bai R, Liu W, Zhao A, Zhao Z, Wang Y, Wang Y, Zhao W and Wang W: miR-22 inhibits osteosarcoma cell proliferation and migration by targeting HMGB1 and inhibiting HMGB1-mediated autophagy. Tumour Biol 35: 7025-7034, 2014. 
15. Rubinsztein DC, Mariño G and Kroemer G: Autophagy and aging. Cell 146: 682-695, 2011.

16. Dong N, Zhu Q, Zhang P, Zhu C, Wang M, Li W, Liu J, Liu Y, Ma B and Wu K: Autophagy downregulates thrombin-induced VSMCs proliferation through lysosomal pathway. Int $\mathrm{J}$ Cardiol 159: 156-158, 2012.

17. Wang HJ, Zhang D, Tan YZ and Li T: Autophagy in endothelial progenitor cells is cytoprotective in hypoxic conditions. Am J Physiol Cell Physiol 304: C617-C626, 2013.

18. Petherick KJ, Williams AC, Lane JD, Ordóñez-Morán P Huelsken J, Collard TJ, Smartt HJ, Batson J, Malik K, Paraskeva C and Greenhough A: Autolysosomal $\beta$-catenin degradation regulates Wnt-autophagy-p62 crosstalk. EMBO J 32: 1903-1916, 2013.

19. Livak KJ and Schmittgen TD: Analysis of relative gene expression data using real-time quantitative PCR and the 2(-Delta Delta C(T)) method. Methods 25: 402-408, 2001.

20. Yin Y,Huang L,Zhao X,Fang Y,Yu S,Zhao Jand Cui B: AMD3100 mobilizes endothelial progenitor cells in mice, but inhibits its biological functions by blocking an autocrine/paracrine regulatory loop of stromal cell derived factor-1 in vitro. J Cardiovasc Pharmacol 50: 61-67, 2007.

21. Wang X, Liu J, Zhen J, Zhang C, Wan Q, Liu G, Wei X, Zhang Y, Wang $\mathrm{Z}$, Han $\mathrm{H}$, et al: Histone deacetylase 4 selectively contributes to podocyte injury in diabetic nephropathy. Kidney Int 86: 712-725, 2014.

22. Ikeda Y, Shirakabe A, Maejima Y, Zhai P, Sciarretta S, Toli J, Nomura M, Mihara K, Egashira K, Ohishi M, et al: Endogenous Drp1 mediates mitochondrial autophagy and protects the heart against energy stress. Circ Res 116: 264-278, 2015.

23. Jiang L, Yin M, Wei X, Liu J, Wang X, Niu C, Kang X, Xu J, Zhou Z, Sun S, et al: Bach1 represses Wnt/ $\beta$-catenin signaling and angiogenesis. Circ Res 117: 364-375, 2015.

24. Lu R, Qu Y, Ge J, Zhang L, Su Z, Pflugfelder SC and Li DQ Transcription factor TCF4 maintains the properties of human corneal epithelial stem cells. Stem Cells 30: 753-761, 2012.

25. Levine B and Kroemer G: Autophagy in the pathogenesis of disease. Cell 132: 27-42, 2008

26. Galluzzi L, Pietrocola F, Levine B and Kroemer G: Metabolic control of autophagy. Cell 159: 1263-1276, 2014
27. Guan JL, Simon AK, Prescott M, Menendez JA, Liu F, Wang F, Wang C, Wolvetang E, Vazquez-Martin A and Zhang J: Autophagy in stem cells. Autophagy 9: 830-849, 2013.

28. Li Y, Zhang L, Zhou J, Luo S, Huang R, Zhao C and Diao A: Nedd4 E3 ubiquitin ligase promotes cell proliferation and autophagy. Cell Prolif 48: 338-347, 2015.

29. Ding Y, Gao H, Zhao L, Wang X and Zheng M: Mitofusin 2-deficiency suppresses cell proliferation through disturbance of autophagy. PLoS One 10: e0121328, 2015.

30. Liu Y and Song L: HMGB1-induced autophagy in Schwann cells promotes neuroblastoma proliferation. Int J Clin Exp Pathol 8: 504-510, 2015.

31. Pei B, Zhao M, Miller BC, Véla JL, Bruinsma MW, Virgin HW and Kronenberg M: Invariant NKT cells require autophagy to coordinate proliferation and survival signals during differentiation. J Immunol 194: 5872-5884, 2015.

32. Cianfanelli V, Fuoco C, Lorente M, Salazar M, Quondamatteo F, Gherardini PF, De Zio D, Nazio F, Antonioli M, D'Orazio M, et al: AMBRA1 links autophagy to cell proliferation and tumorigenesis by promoting c-Myc dephosphorylation and degradation. Nat Cell Biol 17: 20-30, 2015.

33. Lee IH, Cao L, Mostoslavsky R, Lombard DB, Liu J, Bruns NE, Tsokos M, Alt FW and Finkel T: A role for the NAD-dependent deacetylase Sirt1 in the regulation of autophagy. Proc Natl Acad Sci USA 105: 3374-3379, 2008

34. Kageyama S and Komatsu M: Impaired G1-arrest, autophagy, and apoptosis in Atg7-knockout mice. Circ Res 111: 962-964, 2012.

35. Torisu T, Torisu K, Lee IH, Liu J, Malide D, Combs CA, Wu XS, Rovira II, Fergusson MM, Weigert R, et al: Autophagy regulates endothelial cell processing, maturation and secretion of von Willebrand factor. Nat Med 19: 1281-1287, 2013.

36. Thorburn J, Andrysik Z, Staskiewicz L, Gump J, Maycotte P, Oberst A, Green DR, Espinosa JM and Thorburn A: Autophagy controls the kinetics and extent of mitochondrial apoptosis by regulating PUMA levels. Cell Reports 7: 45-52, 2014.

37. Lee IH, Kawai Y, Fergusson MM, Rovira II, Bishop AJ, Motoyama N, Cao L and Finkel T: Atg7 modulates p53 activity to regulate cell cycle and survival during metabolic stress. Science 336: 225-228, 2012 